\title{
Aufsätze
}

\section{Ulrich Wandruszka}

\section{Zur Motivation des französischen Wortakzents}

https://doi.org/10.1515/zrp-2020-0001

\begin{abstract}
Studies of individual languages rarely discuss in any depth the question of how, or to what extent, the positioning of word stress in polysyllabic words is motivated functionally although addressing this issue would mean relating the structural levels of phonetics/phonology, morphology, syntax, semantics and pragmatics systematically to each other. Given that in French practically all (Latin) syllables following the syllable which carries the main stress have been muted, words and word forms are oxytones, generally carrying stress on the last syllable. As a result, endings and suffixes which constitute the morphological head of a word and are, as such, normally unstressed, now also carry stress. This can be seen in faisait or nageur, which in English would correspond to was ${ }^{*}$ doing or

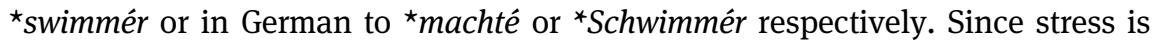
normally positioned on the lexical stem, which carries more information, a morphoprosodic mismatch leading to a countericonic stress pattern obtains in French. Interestingly, this phenomenon has hardly attracted serious attention in linguistics so far, and speakers of French have apparently remained undisturbed by the mismatch. The same is true of the counter-intuitive stress placed on enclitic pronouns in prends-lé! - táke it - nímm(e)s! or vient-íl? - is he cóming? - kómmt er? Apparently, language as an instrument also works when the encoding is suboptimal or dysfunctional. The reason for this must be that language is also supported by the (lexical) content of the signs, which nevertheless makes an appropriate interpretation possible.
\end{abstract}

Keywords: morphological head, word (group) stress, secondary stress, fixed/free stress, falling/rising accent, logical word structure, informational importance, contra-iconic, posttonic syllable

\footnotetext{
Korrespondenzadresse: Prof. Dr. Ulrich Wandruszka, Alpen-Adria-Universität Klagenfurt, Universitätsstraße 65-67, A-9020 Klagenfurt, E-Mail: Ulrich.Wandruszka@aau.at
} 
Schlagwörter: morphologischer Kopf, Wort(gruppen)Akzent, Nebenakzent, fester/freier Akzent, fallender/steigender Akzent, logische Wortstruktur, informationelle Wichtigkeit, kontraikonisch, nachtonige Silbe

\section{Voraussetzungen}

Zumal in einzelsprachlich ausgerichteten Studien zum Wortakzent wird die Frage, wie bzw. inwieweit die Akzentverteilung innerhalb mehrsilbiger Wörter funktionell motiviert ist, meist nicht grundsätzlich erörtert, obgleich mit dieser Frage die Strukturebenen der Phonetik/Phonologie, der Morphologie, der Syntax, der Semantik und Pragmatik systematisch miteinander in Beziehung zu setzen sind. Geht man einmal davon aus, dass die Funktion des (Haupt-)Akzents innerhalb der Domäne des Wortes - wie auch in der des syntaktischen Konstrukts - darin besteht, das informationell jeweils wichtigste Segment als solches zu markieren und hervorzuheben, muss gefragt werden, inwieweit dies für die einzelnen Wortformen mit ihrem aktuellen historisch entwickelten und verfestigten Akzentprofil tatsächlich (noch) zutrifft. Negativenfalls ist zu untersuchen, wie die Dysfunktionalität entstanden ist und welche Kräfte, d.h. welche Entwicklungstendenzen imstande waren, diese natürliche Grundfunktion des Akzents in bestimmten Fällen außer Kraft zu setzen. Bekannte Beispiele (cf. z.B. Meisenburg/Selig 2008, 152) aus Sprachen mit sog. freiem Wortakzent wie span. término 'Termin', termíno ‘ich beende' und terminó 'er/sie beendete' zeigen schnell, dass diese Grundfunktion nicht mehr durchgehend erfüllt sein kann; denn warum sollte die akzentuierte Perfektendung in termin-ó informationell wichtiger sein als die unbetonte Präsensendung in termín-o. Nimmt man an, dass der Verbstamm termin- aufgrund seines spezifischen lexikalischen Inhalts ein größeres informationelles Gewicht aufweist als die abstraktere Personalendung -o (cf. dazu Wandruszka 2011), dann entspricht die stammbetonte Präsensform der angenommenen Funktion des Akzents genau, während das endungsbetonte termin-ó dem klar zuwiderläuft. Diese Akzentuierung ist zwar historisch mit der Silbenstruktur der zugrundeliegenden lateinischen Form terminávit zu erklären, synchronisch betrachtet jedoch ist sie willkürlich und kontraikonisch, gewissermaßen ein morphoprosodischer mismatch. In der Linguistik kann man unter mismatches Phänomene verstehen, in denen sprachliche Merkmale auf verschiedenen Strukturierungsebenen nicht zueinander zu passen scheinen (cf. Pomino/Stark 2011) - man kann in diesem Sinne auch einfach von Diskrepanzen oder Inkongruenzen zwischen Inhalt und Form sprechen.

Die Akzentuierung der Flexionsendung in span. terminó erscheint vor allem auch deswegen als mismatch, weil sie nicht durch irgendwelche anderen, mit 
dem Prinzip der informationellen Wichtigkeit konkurrierenden, phonologischen Regularitäten des Spanischen motiviert ist. In der lateinischen Entsprechung terminávit liegt der Akzent zwar ebenfalls auf der dritten Silbe, was aber durch die allgemeine Silbenstrukturregel des Lateinischen begründet ist, nach der grundsätzlich die schwere Pänultima, hier das lange ā, den Akzent trägt. Mehrsilbige Endungen wie -ấvit sind daher phonologisch regelkonform akzentuiert, einmal abgesehen davon, dass der Themavokal ā noch Teil des Stammes ist. Durch die Reduktion der Auslautsilbe im Altspanischen wird die Verbform dann endbetont, in prinzipiellem Unterschied zum Lateinischen, wo die Ultima von der Betonung allgemein ausgeschlossen und immer als unbetonte Nachtonsilbe erscheint. ${ }^{1}$ Phonologisch regulär hingegen ist im Spanischen die Akzentuierung der Form (yo) termíno, da auf einen Vokal endende Wörter dort tendenziell auf der Pänultima betont sind (im Unterschied zu lat. término und ital. (io) término, daneben freilich auch span. (el) término, cf. supra). Entsprechendes gilt nun für ein französisches passé simple (il) terminá gegenüber dem Präsens (il) termíne mit dem Unterschied, dass hier das Endresultat der Lautentwicklung in beiden Fällen ein Oxytonon ist, da im Französischen mit gewissen Ausnahmen alle Nachtonsilben verstummen.

Auch im Französischen kann folglich der Wortakzent nicht durchgängig die Grundfunktion der informationellen Gewichtung erfüllen, wobei jedoch eine neue relativ einheitliche und einfache Regelung Platz greift: abgesehen von der bedingten Realisierung eines finalen Schwa-Lautes des Typs chambre sind mehrsilbige französische Wörter prinzipiell auf der Ultima betont. Diese Regelung ist nun grundsätzlich nicht mehr intern morphologisch motiviert, weder durch die kommunikative Wichtigkeit noch durch die Silbenstruktur, sondern allein durch die Einheit des Wortes/der Wortform als solcher. Diese bedingungslose Endbetonung, die historisch nicht aus einem Positionswechsel des Akzents resultiert, sondern indirekt aus einer Veränderung der lautlichen Umgebung, eben aus der generellen Reduktion der Nachtonsilben, ist als Tendenz im Französischen so dominant, dass sie gegebenenfalls die Akzentstelle tatsächlich auch versetzt. Dieses lautgeschichtlich eher seltene Phänomen - der Wortakzent verharrt in der Regel konstant auf einer Silbe - lässt sich vor allem bei Proparoxytona beobachten. Da diese jeweils zwei Nachtonsilben haben, wie z.B. lat. frágilis, müssten im Französischen im Fall der Beibehaltung der Akzentstelle beide Silben verstummen. Dadurch entstünde bzw. entsteht ein praktisch allein aus der akzenttragenden Silbe

1 Lateinschüler fallen hingegen gern in die Endbetonung, wenn sie ein Paradigma memorieren, also «cantó, cantás, cantát» etc., wobei aus mnemotechnischen Gründen die Silbe akzentuiert wird, die sich verändert und auf die deshalb das Augenmerk gerichtet werden muss - sie ist in diesem pragmatischen Kontext die wichtigere. 
bestehendes einsilbiges Wort, wie das volkstümlich entwickelte neufranzösische frêle über afz. fraile(s). Wenn aber lat. frágile im 14. Jahrhundert als gelehrtes Wort in das Französische integriert wird, muss der Akzent der zweisilbigen Basis nach rechts rücken, um schließlich die neufranzösische jambische End- und damit Suffixbetonung fragíle zu erreichen. In span./port. frágil hingegen bleibt der Initialakzent bewahrt und das ital. frágile entspricht sogar noch voll dem lateinischen Proparoxytonon. Die deutsche Entlehnung fragíl übernimmt das französische Muster, wie etwa auch in diffizíl ( $\leftarrow$ difficíle) vs. ital. diffícile, span./port. difícil u.a. Daktylische Folgen im Lateinischen werden im Französischen also entweder einsilbig wie frêle oder cálidu $\rightarrow$ chaud, dann auch hómine $\rightarrow$ homme und fráxinu $\rightarrow$ frêne, oder der Akzent wird versetzt wie etwa auch in lat. grácile $\rightarrow$ frz. grêle und gracíle oder cólubra $\rightarrow$ couléuvre gegenüber ital. cólubro (daneben auch colúbro), oder auch fúnebris $\rightarrow$ funèbre u.a. Dieser Zwang zur Akzentverschiebung gilt auch für diverse Entlehnungen aus anderen Sprachen mit fallendem Akzent, wie etwa für zweisilbige englische Nomina agentis des Typs speaker, das zu suffixbetontem speakér mit gerundetem [-œ:R] wird, so wie auch leader $\rightarrow$ leadér.

Von einer semantisch-informationell unangemessenen oder wenn man so will «sinnwidrigen» Betonung der letzten Silbe des französischen Wortes kann man also nicht nur im Fall der Flexionsendungen sprechen, sondern generell auch bezüglich der Wortbildungssuffixe, die ja ebenfalls normalerweise unbetont sind und nicht den Hauptakzent tragen, wie dies z.B. in deverbalem nag-éur oder cour-éur der Fall ist. Dieses Akzentmuster, dem im Deutschen ein abwegiges kontraikonisches ${ }^{*}$ Schwimm-ér/ ${ }^{\star} L a ̈ u f$-ér entspräche, wurde im Laufe der diachronen Entwicklung von den Sprachbenutzern offenbar nicht als störender mismatch empfunden, und auch in der romanistischen Forschung ist es meines Wissens kaum explizit thematisiert worden (cf. infra). Suffixbetonte Ableitungen kommen freilich etwa auch im Spanischen vor, wie gerade in nadadór (aus lat. natatorem) oder corredór. Die Akzentuierung dieser Silbe ist dort allerdings eher systemintegriert als im Französischen, da sie silbenstrukturbedingt regulär im Plural nadadóres und in der femininen Variante nadadóra mit Pänultima-Betonung erscheint. Im Spanischen sind die Nachtonsilben ja keineswegs immer, sondern nur in speziellen lautlichen Umgebungen verstummt, so dass dort im Unterschied zum Französischen das trochäische fallende Akzentmuster grundsätzlich erhalten bleibt. Endbetont sind im Spanischen auch Ableitungen wie natación/nataciónes oder ingratitúd, sinceridâd und in der Flexion sämtliche Infinitivendungen wie in nad-ấr, com-ér etc., sowie die Futurformen mandaré/mandarâs, entsprechend ital. manderò/manderai. Es gibt im Spanischen also differenzierte Wortbetonungsregeln, die auf die jeweilige lautliche Umgebung Bezug nehmen, wie z.B. die, dass auf einen Vokal oder die Konsonanten $n / s$ auslautende Wörter im un- 
markierten Fall Paroxytona sind und dass in Wörtern, die auf einen Konsonanten außer $n / s$ enden, die Ultima akzentuiert ist. Im Italienischen hingegen finden wir in diesen Fällen ein auslautendes - $e$ wie in: nuotatore/-ori, corridore und natazione/-ioni, ingratitudine oder dann nuotare, vedere, dormire etc., die weitgehend dem lateinischen bzw. dem vulgärlateinischen Silbenstand entsprechen. Endbetonung findet man in Ableitungen wie sincer-ità, qual-ità und dergleichen mit verstummtem intervokalischen - $t$ - im Lateinischen. Auf weitere syn- oder diachrone romanische Details und Differenzen soll hier jedoch nicht eingegangen werden.

Die unmotivierte sinnwidrige Betonung der französischen Endungen und Suffixe wird deswegen nicht als störend empfunden, weil mit der Akzentuierung nicht das Endungsmorphem als solches gemeint ist, sondern einfach nur die letzte Silbe des Wortes. Da diese prinzipiell akzentuiert ist, liegt der Akzentuierung keine Intention auf der Basis einer freien Wahlmöglichkeit zugrunde und hat damit auch keine semiotische Relevanz. In diese Richtung zielt schon Pierre Guiraud (1980, 118-119) mit seinem Hinweis auf die

«situation illogique qui fait porter l'accent sur une détermination secondaire et qui trouble la structuration du système. Car l'accentuation oxytonique du suffixe est une des causes qui contribue au caractère arbitraire du mot français en affaiblissant le sentiment de la dérivation; dans des mots comme vérité, blancheur etc. le suffixe accentué n'est pas senti comme un morphème, ni la forme comme un dérivé».

Auch wenn das Prädikat «arbitraire» für Suffixableitungen wie blancheur oder nageur etc., einfach aufgrund ihrer Bedeutung und morphologischen Durchsichtigkeit, überzogen ist, kann man annehmen, dass ein betontes Suffix im Französischen deswegen nicht «auffällt» und stört, weil es der normalen Wortbetonung entspricht. Wie bereits angemerkt ist der französische Wortakzent inhaltlich grundsätzlich nicht motiviert, einmal abgesehen von seiner delimitativen Funktion, durch die das Ende des Wortes bzw. des mot phonique (cf. infra) markiert wird. Aus diesem Grund stören auch die anderen im Französischen allgegenwärtigen «falschen» bzw. nicht intendierten Betonungen, auf die wir noch zurückkommen werden, nicht wirklich.

\section{Akzentmuster und logische Struktur des Wortes}

Geht man also davon aus, dass der Hauptakzent polymorphemischer bzw. mehrsilbiger Wörter natürlicherweise auf die Silbe mit der höchsten informationellen Wichtigkeit $\mathrm{zu}$ liegen kommt, sind unterschiedliche Arten von Wichtigkeit anzunehmen; denn keines der interdependenten Bestandteile eines Wortes kann in jeder Hinsicht unwichtig sein. Wir unterscheiden die informationelle Wichtigkeit, 
womit die relative Menge an neuer nicht zu erwartender Information gemeint ist, von der strukturellen Wichtigkeit, die für die grammatische Struktur und syntaktische Kategorie eines Ausdrucks von Bedeutung ist (cf. Wandruszka 2011 und 2015). Der die Kategorie eines Ausdrucks bestimmende Bestandteil ist dessen Kopf-(Funktor), während der jeweils andere als Komplement bzw. Attribut fungiert, das den Kopf determiniert und inhaltlich spezifiziert. Ersterer ist in der Regel (cf. unten) ein Funktionselement, wie eben eine Flexionsendung oder ein Wortbildungssuffix, und Letzterer normalerweise der lexikalische Kern eines komplexen Wortes oder auch ein Präfix.

Der kategoriebestimmende Kopf steht sowohl für die Wortart als auch für die syntaktische Funktion eines Ausdrucks. In einer Form wie chant-ons identifiziert die Endung den Ausdruck als Verbum und dessen funktionale Kategorie als Prädikat oder Satz, im Unterschied etwa zur Verbform chant-ant mit der Funktion des Attributes eines Nomens wie in accent chantant, im Unterschied auch zu einer Ableitung wie chant-eur, wo die Endung den Ausdruck als Nomen kennzeichnet, das nun nicht mehr der Wortart Verbum angehört und somit kein direktes Objekt zu sich nehmen aber von einem Adjektiv determiniert und mit einem Artikel verbunden werden kann, wie in un chanteur allemand. Insofern sind die Endungen und das Suffix von entscheidender struktureller Wichtigkeit, während der Stamm chant- nicht notwendigerweise ein Indiz für die Wortart oder die syntaktische Funktion des Gesamtausdrucks ist. Dies illustriert einerseits eben das deverbale Nomen chanteur und andererseits etwa ein deadjektivisches Nomen wie lent-eur bzw. ein denominales Verbum wie huil-er ‘ölen'. Der Stamm bezeichnet indessen den Vorgang, die Eigenschaft oder das Objekt, d.h. die Entität, mit der das Denotat des Gesamtausdrucks etwas zu tun hat.

Der Stamm repräsentiert jeweils das spezielle lexikalische Individuum als Element einer theoretisch unendlichen Menge, während die Endung die abstraktere allgemeine Kategorie und Funktion des Ausdrucks markiert und damit einen deutlich geringeren Mitteilungswert besitzt. Ein Wert, der sich zudem dadurch reduziert, dass Kategorie und Funktion eines Ausdrucks normalerweise in den jeweils vorausgehenden sprachlichen Einheiten antizipiert sind. So ist in einem Satz wie Demain il partira die Kategorie Verbum, dessen Finitheit und die Futurität, also Person und Tempus, im Unterschied zur lexikalischen Bedeutung des Stammes, eindeutig vorhersehbar, womit die entsprechenden Trägermorpheme, d.h. die Flexionsendungen, praktisch keine neue Information mehr für den Empfänger enthalten. ${ }^{2}$ Eben deswegen können die Endungen im Französischen, so-

2 Daher kann etwa im Kreolischen einfach ein endungsloser Stamm in Verbindung mit dem Subjektpronomen als Verbform verwendet werden: mwe sav «je sais» (Haiti). 
weit sie nicht den Akzent tragen, vollständig verstummen, so dass die Verbform, morphologisch «kopflos», (lautlich) allein durch den Stamm repräsentiert wird: je/tu/il(s)/on/les enfants mange(nt) gegenüber nous mangeons/vous mangez.

Normalerweise trägt also der als Komplement/Determinans des flexivischen oder affixalen Kopffunktors fungierende Stamm den Hauptakzent, während der Funktor als die weniger betonte Vor- oder Nachtonsilbe erscheint - als Vortonsilbe bei Postdetermination, wie etwa in frz. dedâns oder dt. hin-únter und als Nachtonsilbe bei Prädetermination mit fallendem Akzentmuster, wie in dt. Schwímmer, síngen und ital./span./port. cânta, lat. cântor oder proparoxyton ital. cántano. Die Tatsache, dass die Endungen und Affixe als kategoriebestimmende Köpfe natürlicherweise den weniger betonten Bestandteil bilden, kann andersherum auch als prima-facie-Kriterium für die theoretisch nicht immer ganz einfache Bestimmung des Kopfes eines mehrgliedrigen Ausdrucks verwendet werden. Diese Korrelation besteht aber, wie gesagt, in den romanischen Sprachen nicht mehr durchgehend und im Französischen nach dem Verstummen der lateinischen Nachtonsilben überhaupt nicht mehr, weswegen wir diesbezüglich von mismatch sprechen.

Phonetisch erklärt sich dieses Verstummen damit, dass Nachtonsilben generell besonders schwach ausgeprägt sind und daher auch Funktoren in dieser Position, nach einer klitischen Phase, in hohem Maße reduktionsanfällig sind (cf. Wandruszka 1992). Durch ein trochäisch fallendes Akzentmuster charakterisierte prädeterminierende (OV-)Sprachen mit ausgeprägter Endungs- und Suffixmorphologie tragen somit den Keim ihrer Auflösung, d.h. ihres typologischen Wandels bereits in sich. Generell ist ein lautlicher Schrumpfungsprozess zu beobachten, der in bestimmten Konstellationen zu einer Verkehrung des typologisch bedingten Wortakzent-Grundmusters einer Sprache führen kann, in unserem Fall von einem fallenden zu einem steigenden jambischen Betonungsmuster. Während zweisilbige Paroxytona mit einsilbiger Endung schlicht einsilbig werden, wie múru $\rightarrow$ mur, vénit $\rightarrow$ vient, entstehen aus drei- und mehrsilbigen Paroxytona Oxytona, die mit der akzentuierten Silbe enden, wie in murâle $\rightarrow$ murál oder monuméntum $\rightarrow$ monumént, cantấvit $\rightarrow$ chantấ, womit man auch innerhalb des Wortes $\mathrm{zu}$ einem für postdeterminierende Sprachen charakteristischen jambischen Akzentmuster gelangt. Aus den zweisilbigen lateinischen Suffixen/Endungen mit fallendem Akzent entstehen dabei die kontraikonisch akzentuierten einsilbigen Suffixe und Endungen des Französischen. Dadurch erhält das Französische eine Vielzahl von Wörtern/Wortformen mit betontem vokalischem Auslaut, während unbetonte Vokale am Wortende, außer Schwa, im Prinzip nicht mehr vorkommen.

Ab dem Zeitpunkt des völligen Verlustes der Nachtonsilben - im Französischen möglicherweise durch den Einfluss des dynamischen germanischen Ak- 
zents mit seiner markanten Differenz zwischen betonter und unbetonter Silbe (cf. Geisler, 1982) - gibt es praktisch keinen fallenden Akzent mehr, denn auf eine akzentuierte Silbe kann nun im Prinzip nichts mehr folgen: ... s-ś-s $\rightarrow$... s-ś. Die konsequente Endbetonung der französischen Wörter ist historisch vor allem dadurch bedingt, dass das Lateinische zu den sog. gewichtssensitiven Sprachen gehört, bei denen zur Bestimmung der Akzentsilbe auch das Silbengewicht eine Rolle spielt (Meisenburg/Selig 2008, 151-152). Im Unterschied zum Deutschen fällt der Akzent im Lateinischen bei entsprechendem Silbengewicht immer auf die Pänultima und rückt daher bei Suffixableitung jeweils um eine oder mehrere Silben nach rechts: páter $\rightarrow$ patérnus $\rightarrow$ paternális $\rightarrow$ paternalitátem, was schließlich zur französischen Oxytonie führt: père $\rightarrow$ patérne $\rightarrow$ paternél $\rightarrow$ paternalité. Im Deutschen hingegen ist der wortinitiale Akzent hier unverrückbar: Vâter $\rightarrow$ väterlich $\rightarrow$ Väterlichkeit $\rightarrow$ väterlicherseits.

Da das Ende bzw. die letzte Silbe eines Wortes nun aber per se keine akzentogene Größe darstellt, gibt es für die Position des neufranzösischen Wortakzents keine direkte (mor)phonologische Motivation. Eine indirekte Motivation oder Regel, auch für den Sprecher, kann aber einfach in dem Umstand gesehen werden, dass mit dem Ausschluss fallender Akzentmuster jedes Wort auf eine betonte Silbe enden muss bzw. aus einer betonten Silbe besteht. Dies gilt tendenziell auch für Wörter mit auslautendem Schwa bzw. $e$-muet wie faible, concombre, quatre oder signe. Da die neufranzösischen Verhältnisse auf historische Silbenstrukturbedingungen zurückgehen, wie eben die Akzentuierung der schweren Pänultima im Lateinischen und das spätere zur Oxytonie führende Verstummen der Nachtonsilben, sind darüber hinaus für den neufranzösischen Wortakzent (wie für den des sog. mot phonique, s.u.) keine weiteren synchronen phonologischen Regeln mehr zu formulieren.

Das steigende jambische Akzentmuster ist auch in der wortübergreifenden Syntax charakteristisch für postdeterminierende Sprachen mit dem Akzent auf dem nachgestellten Determinans, also dem Adjektiv in voiture rouge, dem Adverb in parler lentement, der NP in derrière la maison oder dem Nomen in la maison. In dem diesbezüglich strikten Französisch gibt es daher keine betonten Determinantien in Voranstellung mehr und keine unbetonten Determinantien in Postposition, etwa nach Art der enklitischen Objektpronomina im Italienischen oder Spanischen wie in farlo, dartelo, vedendoti und dergleichen. Infolge der Generalisierung des jambischen Akzentmusters im Französischen müssen derartige Klitika dem verbalen Kopf vorangehen: pour le faire, pour te le donner, en te voyant. Hier ergibt sich folglich ein «klassischer» Konflikt zwischen der Tendenz zur Postdetermination, d.h. zur VO-Folge einerseits, und dem Verlust des fallenden trochäischen Akzents andererseits - ein Konflikt, der im Französischen im Unterschied zu anderen romanischen Sprachen ausnahmslos zugunsten des Akzent- 
musters und zu Lasten der typkonformen Determinationsrichtung gelöst wird. Wenn das Französische hier $\mathrm{OV}$-Strukturen aufweist, dann eben nicht, wie mitunter behauptet wurde (cf. z.B. Sasse 1977), weil es in Resten noch, wie das Lateinische, eine $O V$-Sprache wäre, so wie das Deutsche in Strukturen der Art ... dass Maria ihn besucht/um es zu tun (oder auch nominal in ... dass Maria einen Fréund besucht), sondern weil es als besonders ausgeprägte $V O$-Sprache keinen fallenden Akzent mehr duldet.

Aus diesem Grund ist andererseits die Akzentuierung des Objektpronomens in frz. prends-lé nicht darauf zurückzuführen, dass es ein (betontes) Determinans ist, sondern allein darauf, dass es die letzte Silbe bildet. Prends-le nimmt hier also die Gestalt eines oxytonen Wortes an, dessen Endsilbe freilich sinnwidrig akzentuiert ist, da sie pragmatisch nicht etwa kontrastiv betont werden soll. Prends-lé entspricht nicht einem akzentuell analogen dt. nimm îhn, sondern einem normalen unmarkierten nímm ihn/es mit enklitischem Pronomen. Le ['lœ] ist folglich ein akzentuiertes Enklitikon und als solches ein in sich widersprüchliches Gebilde, ein mismatch im eigentlichen Sinn des Wortes, prinzipiell vergleichbar dem akzentuierten Suffix des Nomens nagéur. Die Betonung des Enklitikons ist eben nur deshalb möglich, weil es als letzte Silbe eines Wortes prends-le, das als solches im Französischen regulär oxyton ist, interpretiert wird. ${ }^{3}$ Außerhalb eines solchen morphonologischen Kontextes, also etwa in proklitischer Position, ist ein Klitikon nicht akzentuierbar, da der ihm inhärente satzphonetische Status dort nicht aufgehoben werden kann. Wenn also der Imperativ unmittelbar mit dem Verbum einsetzen soll, muss entweder gegen die Unzulässigkeit fallender Akzentmuster im Französischen verstoßen werden ( ${ }^{*}$ prénds-le) oder gegen die Unbetonbarkeit von Klitika bzw. gegen eine inhaltlich nicht-gegebene und nicht-gemeinte (kontrastive) Betonung des Pronomens. Im zweiten Fall verliert das Pronomen nun zwar phonetisch seinen Status als Klitikon, wird aber dennoch als solches verstanden, wobei die Betonung interessanterweise einfach überhört wird. Man sieht unbewusst davon ab und perzipiert das Pronomen in seiner eigentlichen pragmatischen Funktion als vorerwähntes thematisches Element, das aber als letzte Silbe eines Wortes den Akzent übernehmen muss.

Die Klitika werden also gemäß der dominanten Akzentstruktur französischer Wörter in der Enklise akzentuiert, wobei der Konflikt zwischen einer inhaltlichpragmatischen Motivation und einer solchen des (Wort-)Akzentmusters zugunsten der Letzteren entschieden wird. Die Interpretation der Folge Verbum-Pro-

3 Die besonders enge Bindung enklitischer Morpheme an ihre Bezugseinheit zeigt sich auch in der Zusammenschreibung und Univerbierung italienischer Kombinationen wie farlo/fallo/dimmi und entsprechend im Spanischen oder Portugiesischen. So ist auch der Bindestrich in frz.prends-le! etc. motiviert. 
nomen als komplexes Wort wird dadurch befördert, dass das Pronomen als «ursprüngliches» Klitikon sehr eng mit seiner lexikalischen Bezugseinheit verbunden ist, was in besonderem Maß - wie bereits erwähnt - für nachtonige enklitische Elemente gilt (cf. Wandruszka 1992). Die Entwicklung zur Entklitisierung und Akzentuierung datiert übrigens erst seit dem Mittelfranzösischen, denn das Objektpronomen konnte bis zur Renaissance auch in einer derartigen Position seinen klitischen Status bei fallendem Akzent beibehalten, wie Maurice Grevisse (1975, 461) anmerkt: «le pronom le, après un impératif sans négation, pouvait être inaccentué: perd-LE, par exemple, pouvait rimer avec perle».

Wo es eine betonte Variante des Objektpronomens gibt, wird diese in unserer Position verwendet, wie etwa in crois-moi! oder approche-toi!. In solchen Fällen besteht der mismatch nur zwischen der formal prinzipiell korrekten (und obligatorischen) Akzentuierung des Pronomens und der nicht-intendierten Betonung, im Unterschied etwa zu einer Präpositionalphrase pour toi. Dasselbe gilt für ein approchez-vous!, dessen Pronomen formal jedenfalls korrekt akzentuierbar ist, hier aber akzentuiert werden muss, auch wenn es nicht betont werden soll. Im Italienischen wird in diesem Kontext gerade die klitische Variante ti und nicht die betonte te verwendet, also z.B. avvicinati! und credimi (a me)!, entsprechend auch im Spanischen: créeme (a mî)! und acércate!.

Eine inhaltlich widersinnige bzw. nicht-intendierte Versprachlichung, nämlich die Akzentuierung von nicht $\mathrm{zu}$ Betonendem und eigentlich nicht Betonbarem liegt auch in der Inversionsfrage mit pronominalem Subjekt wie in vient-íl? vor, womit ebenfalls kein kontrastives kommt ér gemeint ist, sondern unmarkiertes kómmt er? mit enklitischem Subjekt, so wie etwa auch in einem qu'elle heure est-îl? mit seinem nicht-referentiellen und daher im Prinzip absolut unbetonbaren Pronomen. Auch diese Unstimmigkeiten sind überhörbar, weil die Akzentuierung des dem Verb folgenden Pronomens gemäß den neufranzösischen Intonationsregeln obligatorisch ist und daher nicht als intendierter, einer freien Wahl entsprungener Zeichenwert interpretiert wird.

Ebenfalls nur in enklitischer Position und nicht etwa in Isolation kann das Subjektpronomen der 2. Pers. Sing. den Akzent des Wortes bzw. des mot phonique tragen, wie in einem où vas-tú?, statt der betonten Variante wie z.B. in c'est toi. Die Frequenz des konkurrierenden où tu vas? mit proklitischem Pronomen ist freilich heute schon bedeutend höher, was entsprechend auch für où va-t-on? gegenüber où on va? gilt oder für que fera-t-on? vs. qu'est-ce qu'on fera. Auch die Pronominaladverbien en und $y$ sind in Postposition akzentuiert: prenez-en! oder penses-y! (mit eingefügtem [z] wie in vas-y!). Nur das Pronomen der 1. Pers. Sing. bleibt in Postposition klitisch tonlos: puís-je?, ai-je, suis-je und pourrais-je vs. pourrais-tú. Auffällig sind auch literarische Formen wie dussé-je, wo das $e$-muet der Endung von dusse silbisch und akzentuiert wird, damit das klitische je daran 
angelehnt werden kann. Klitisch bleibt in der Position nach dem Verbum auch das neutrale Demonstrativpronomen ce, wie z.B. in qui ést-ce? - wer íst das?, im Unterschied zu qui est-îl?. Wie je in puis-je kann ce in est-ce als nicht-silbischer frikativer Auslautkonsonant artikuliert werden [ki 'æs] und insoweit ebenfalls als Teil des Bezugswortes erscheinen. Die beiden Pronomina sind hier also keine nachtonigen Enklitika mehr und somit auch keine Ausnahme von der allgemeinen Regel, die derartige fallende Akzentmuster im Neufranzösischen ausschließt. Auf der Basis dieser Zusammenhänge lässt sich nun auch das Faktum erklären, warum die Inversionsfrage im Neufranzösischen nur noch mit pronominalem Subjekt akzeptabel ist, wie in vient-íl? oder pourquoi pars-tú?, und seit dem Mittelfranzösischen nicht mehr mit nominalem Subjekt, wie in part Michel? oder part votre père?. Da das Subjekt in Sätzen wie vient-íl?/pars-tú? pragmatisch gerade nicht betont ist bzw. nicht betont sein kann und daher einem dt. kómmt er/géhst $d u$ ? und nicht etwa einem kontrastiven kommt ér/gehst dú? entspricht, müsste die nominale Ausführung - dt. kómmt/géht Michael? - eine im Französischen inakzeptable fallende Intonation aufweisen, nämlich eben párt Michel? oder párt votre père?, statt votre père, part-il?. Diese kommunikative Struktur mit nachgestelltem Subjekt kann aus den genannten Gründen nur durch ein pronominales part-íl? wiedergegeben werden, während ein nominales Subjekt in dieser Position auch inhaltlich-pragmatisch betont wäre: vient/part Michél, was heute aber durch ein est-ce/ c'est Michel qui part? bzw. pronominal est-ce/c'est lui qui part? dargestellt wird.

\section{Das Begriffspaar Post-/Prädetermination in der deutschen Romanistik}

Theoriegeschichtlich bemerkenswert ist die inkonsistente Verwendung des zentralen Begriffs der 'Determination' in der (Morpho-)Syntax bei deutschen Romanisten, die eine gewisse Verwirrung gestiftet hat und dies offensichtlich bis heute tut (cf. Wandruszka 1980). So spricht etwa Kurt Baldinger (1968) von einem strukturellen Wandel von der lateinischen Postdetermination zur neufranzösischen Prädetermination. Als Zeugen für diesen Wandel führt Baldinger u.a. folgende Phänomene an: Die syntaktische Funktion, Numerus etc. des Nomens werden durch Artikel in Verbindung mit einer Präposition markiert statt durch eine Endung. Ersetzung des suffixalen Komparativs durch Gradadverbien und des Diminutivsuffixes -ette durch vorangestelltes Adjektiv petit. Ersetzung der Personalendungen beim Verbum durch ein obligatorisches Subjektpronomen und die Ersetzung des passé simple und des Futurs durch periphrastische Formen, wie il a mangé und il va manger. Ersetzung des attributiven Partizips durch eine Relativ- 
konstruktion, wie in la vache qui rit statt la vache riante. Ersetzung der Inversionsfrage durch die Fragekonstruktion mit est-ce que. Als nicht ganz erklärliche Ausnahme erscheint ihm hingegen die zweigliedrige Negation mit nachgestelltem pas in elle ne mange pas, auch ohne vorangestelltes ne in je (ne) sais pas (cf. infra).

Hier ist Determination offensichtlich nicht in einem logisch-syntaktischen Sinn gemeint, d.h. als Relation zwischen Determinatum (Operand) und Determinans (Operator). Gemeint ist vielmehr Determination in einem morphologisch-paradigmatischen Sinn: «Was determiniert, muss Morphem und bound form sein» (Wandruszka 1980, 91). Diese charakteristische Struktur des Neufranzösischen hat Harald Weinrich (1966) mit folgender deskriptiven Formulierung zusammengefasst (sinngemäß): Für das Nfz. gilt tendenziell: Morphem vor Lexem. So stehen im Neufranzösischen tendenziell gerade die Elemente voran, die ein finites grammatisches Paradigma bilden und den Einheiten, auf die sie sich beziehen, eine bestimmte Position innerhalb dieses Paradigmas und damit bestimmte Funktionen und Merkmale zuweisen (cf. Wandruszka 1980, 58). ${ }^{4}$ Dies sind nun aber eben die Elemente, die als klassen- oder kategoriebestimmende Konstituenten in unserem Sinn den determinierten Funktorkopf eines komplexen Ausdrucks darstellen und daher gerade für die postdeterminierende Struktur des Neufranzösischen stehen, wie z.B. die Präposition oder das finite Auxiliar in periphrastischen Verbformen.

Betrachtet man das jeweils akzentuierte Glied eines komplexen Ausdrucks als dessen Determinans, wie etwa in maison blanche oder maison mère, müsste man sowohl im Fall der Endungen (außer im Französischen) als auch in dem der Präposition oder des Auxiliars - wenn man diese mit Baldinger oder Geisler (1982) als Determinantien definiert - eine Ausnahme von dieser Konstante ansetzen, da diese Elemente ja gerade unbetont sind. Geisler (ib., 97) sieht sich daher veranlasst, eine Unterscheidung zwischen lexikalischer und morphologischer Determination zu treffen:

«Im Gegensatz zur lexikalischen Determination [Nomen-Adjektiv und dergleichen] trägt bei morphologischer Determination nicht das Determinans den Hauptakzent, sondern genau umgekehrt das Determinatum, während die Determinantien unbetont sind (was gleichzeitig Voraussetzung für die Klitisierung bei Ausbildung der Morpheme ist)». ${ }^{5}$

4 So etwa noch bei Stefan Barme (2012, 18), der davon spricht, dass bei den Verben «die Markierung der grammatischen Kategorie Person in der geschriebenen Sprache überwiegend prä- und postdeterminierend erfolgt» - d.h. durch Subjektpronomen und Verbalendung. Hinzukommt, dass zwischen Subjekt und Verbum im logischen Sinn gar keine Determinations- sondern eine Prädikationsrelation besteht.

5 In diesem Sinn auch Weinrich (1976, 227), der von der Grundstruktur der französischen Sprache spricht, «nach der in der Regel die determinierenden Morpheme vor und die determinierenden Lexeme hinter dem Substantiv stehen». 
Als Beispiele hierfür werden lat. pátrem und dt. Kíndheit, spársam oder spáren genannt, deren Endungen und Suffixe als Determinantien interpretiert werden. Die Annahme einer solchen Umkehrung erscheint freilich nicht plausibel, da das die Determinationsverhältnisse widerspiegelnde Akzentmuster nicht von der morphologischen Gestalt der Konstituenten abhängig ist und sich prinzipiell einheitlich darstellen sollte. Gerade Suffixe sind in der Regel eindeutig als determinierte Köpfe zu identifizieren (cf. supra), da sie die Träger der Kategorie des Gesamtausdrucks sind, also in Kindheit der Merkmale [Nomen, Femininum, Singular]. Darüber hinaus auch semantisch, da das Wort nicht ein Kind bezeichnet, sondern eine Lebens- und Entwicklungsphase, wofür das Suffix -heit steht. In sparsam steht das Suffix für die Wortart, denn es macht als Kopffunktor mit einem Verbstamm spar- ein Adjektiv. Im Fall von Flexionsendungen wie bei patr-em ist die Situation vielleicht weniger eindeutig aber auch hier ist die Endung klassenbestimmender Kopf, der das Wort als singularisches Nomen im Akkusativ kategorisiert. Offenbar ist der Begriff Kopf bei Baldinger und Geisler aber auch bei Weinrich und verschiedenen anderen Romanisten einfach mit der Vorstellung eines selbständigen Vollwortes bzw. des lexikalischen Kerns eines mehrgliedrigen Wortes verbunden, so dass eine Präposition oder gar ein Suffix bzw. eine Endung nicht als Kopf in Erwägung gezogen werden.

Es gibt dort also keine Umkehrung der Akzentmusters und damit keinen mismatch in Gestalt eines betonten Determinatums, da eben auch in diesen Fällen weiterhin das Determinans, hier in Gestalt des lexikalischen Kerns, den Hauptakzent trägt. Es ist auch nicht so, wie Geisler schreibt, dass die lateinische Syntax prädeterminierend wäre, also $\mathrm{O}-\mathrm{V}$, und die Morphologie postdeterminierend und sich daher «lexikalische und morphologische Determination in der Linearisierungsrichtung unterscheiden» (Geisler 1982, 53). Die reiche Endungs- und Suffixmorphologie des Lateinischen ist vielmehr gerade ein Indiz für seine prädeterminierende Grundstruktur mit trochäischem Akzentmuster. Hier offenbart sich ein tatsächlicher Nachteil eines solchen Determinationsbegriffs, mit dem die strukturelle Isomorphie zwischen «lexikalischer» und «morphologischer» Determination, d.h. die Parallelität von freier Syntax und Morphosyntax nicht nur nicht zu erfassen ist, sondern direkt verschleiert wird. Das eigentliche Problem dieser Terminologie ist aber darin $\mathrm{zu}$ sehen, dass bei der Unterscheidung von «lexikalischer» und «morphologischer» Determination der Begriff Determination nicht einheitlich verwendet wird: im ersten Fall nämlich im logischen Sinn, z.B. in maison blanche, und im zweiten Fall, z.B. in Kindheit, im paradigmatischen Sinn, da das Suffix -heit den Stamm Kind- ja nicht determiniert.

Als weitere Kronzeugen für französische Prädetermination werden Präpositionalphrasen wie par le livre (Geisler 1982, 214, und Baldinger 1968, 92ss.) aufgeführt, wiewohl die Präposition hier eindeutig als Kopf der Struktur fungiert, 
was eben mit ihrer Unbetontheit übereinstimmt. Als prä- und postdeterminierend wird die periphrastische Verbform j'ai chanté gewertet, wobei sich ersteres auf das Verhältnis von Auxiliar und Partizip bezieht, das aber zweifellos postdeterminierend ist; denn das kategoriebestimmende Auxiliar wird von dem Partizip, das von diesem strukturell abhängt, näher spezifiziert und nicht umgekehrt. Als postdeterminierend werden sowohl Suffixableitungen wie die Nomina fad-eur und popre-té, als auch die Verbformen chant-ais und chant-ons!, sowie die Folge sais pas betrachtet. Letzteres ist nun insofern interessant, als die nachgestellte Negationspartikel pas - was meist übersehen wird - wie z.B. ein Adverb tatsächlich ein Determinans (des Prädikats) ist und als solches funktionsgerecht den Akzent trägt, im Unterschied zu den Funktorköpfen -ais und -ons der beiden Verbformen, die den angesprochenen prosodischen mismatch zwischen Betonung und Funktion aufweisen. Im Französischen ist es ja gerade nicht so, dass das nachgestellte morphologische «Determinans» im Sinne der Autoren unbetont wäre und der Hauptakzent auf dem Stamm läge. Wie wir gesehen haben, ist der Grund dafür in externen Faktoren wie eben dem Verstummen der nachtonigen Auslautsilben im Fall der französischen Suffixe und Endungen zu finden.

Aufgrund der Linearität und Eindimensionalität des sprachlichen Mediums ist der Konflikt zwischen Determinationsrichtung und Akzentuierung systemimmanent und nicht auflösbar, weil gewisse Determinantien/Komplemente, zumal pronominale und klitische, grundsätzlich unbetont sind und daher nur «verkehrt» stehen können, jedenfalls wenn ein Akzentmuster so dominant ist wie das jambische im Französischen. Die Voranstellung in einem le faire verstößt gegen die Determinationsrichtung und die Nachstellung in *faire le gegen den verbindlichen Finalakzent. Entsprechendes gilt natürlich für prädeterminierende Sprachen mit trochäischem Akzentmuster, in denen die Voranstellung, wie etwa in dt. dass Maria ihn besúcht, gegen die Initialbetonung eines fallenden Akzents, wie in ... einen Fréund besucht, verstößt, und die Nachstellung in einem ... *dass Maria besucht ihn gegen die Determinationsrichtung. Es ist freilich zu vermerken, dass der deutsche Hauptsatz hinsichtlich der Position (nicht der Reihenfolge) der Objekte zum Verbum postdeterminierend ist, so dass in diesem Fall mit einem Maria besucht ihn gegen das jambische Akzentmuster verstoßen wird, das bei nominalen Objekten gegeben ist: Maria besucht einen Fréund. Zusammenfassend lässt sich also festhalten, dass verbabhängige Pronominalobjekte weder in prä- noch in postdeterminierenden Sprachen systemkonform «korrekt» serialisiert werden können. Da das determinierende Glied im unmarkierten Fall den Akzent trägt, sind unbetonte bzw. klitische Determinantien diesbezüglich in sich widersprüchliche Entitäten - eben Stellvertreter, die keine neue Information vermitteln. Oder unter anderem Aspekt betrachtet: unbetonte Determinantien bewirken automatisch eine Akzentverlagerung auf das jeweilige Determinatum, den jeweiligen 
Kopf, also auf die Konstituente, die normalerweise gerade unbetont ist: dt. síeht ihn/ihn síeht und frz. le vóit.

Die meisten der hier betrachteten Sprachen sind freilich nicht so konsequent einem Akzentmuster verpflichtet wie das Französische. Im Deutschen können unbetonte Objektpronomina enklitisch und proklitisch zum Verbum auftreten, wie in den genannten deutschen Beispielen (besúcht ihn/ihn besúcht), wobei freilich postdeterminierender Hauptsatz und prädeterminierender Nebensatz, wie gesagt, unterschiedlichen Serialisierungstypen angehören. Im Gegensatz zu den Pronomina stehen betonte nominale Objekte im Deutschen immer «richtig», d.h. systemkonform: steigend postdeterminierend im VO-Hauptsatz, Maria besucht einen Fréund und prädeterminierend fallend im OV-Nebensatz, dass Maria einen Fréund besucht. Was die Pronominalobjekte angeht, kann man auch einfach sagen, dass sie im Deutschen, im Unterschied zum Französischen, immer dort stehen, wo auch die Nominalobjekte auftreten, wobei das Akzentmuster nicht von Relevanz ist.

Wie wir gesehen haben, verhält es sich in den romanischen Sprachen und zumal im Französischen gerade nicht so. Gemäß dem zum Determinationstyp passenden Akzentmuster, in unserem Fall dem jambischen, geht das unbetonte klitische Objektpronomen im Unterschied zum nominalen dem Verbum voran. Entsprechendes gilt für die Pronominaladverbien wie z.B. ital. ci und ne in einem non ci vado mai. Die Folge Pronomen-Verbum, wie in Marie le voit, verbindet mit dem steigenden Akzentmuster auch die natürliche Reihung Thematisches vor Rhematischem. Das betonte Pronomen steht entsprechend nach dem Verbum wie typisch in span. te quiere a ti bzw. ital. pensa a te, ama te und frz. il pense à toi oder das bereits angesprochene imperativische assieds-toi! Romanische Objektpronomina wie lo oder le(s)/la, frz. te sind als echte Klitika nicht betonbar im Gegensatz zu den deutschen ihn, sie, dich etc. in Maria liebt íhn/mích/dích. Ausnahmen bilden lediglich die französischen Objektpronomina le(s)/la in imperativischem prends-le/la! Im Unterschied zum Deutschen also ist der Akzent in den romanischen Sprachen für die Position von Pronomina relevant, womit Enklitika und damit ein fallender Akzent verhindert werden. Variation findet sich allerdings in den südromanischen Sprachen mit der Möglichkeit einer enklitischen Position nach infiniten Formen des Verbums, wie in ital. non posso farlo gegenüber non lo posso fare. Wie erwähnt gibt es in diesen Sprachen auch innerhalb des Wortes sowohl steigende als auch vor allem fallende Akzentmuster, mehrheitlich Paroxytona (cf. u.a. Christine Kaschny 2011), also cantó vs. canto/cantano etc. Wir werden darauf zurückkommen. 


\section{Fallender Akzent im Wortinneren und in der Satzsyntax}

Der Konflikt zwischen Akzentmuster und Determinationstyp ist im Französischen allgegenwärtig, tritt aber, wie gesagt, auch im Deutschen auf, sowohl in Konstruktionen der freien Syntax als auch im Wortinneren. Morphosemantische mismatches, wie wir sie genannt haben, finden sich in deutschen Wortbildungsprodukten mit prädeterminierenden nicht-betonten Elementen, wie z.B. in bestimmten Präfixableitungen. Wenn Präfixe als determinierende Attribute fungieren, sollten sie entsprechend auch den Hauptakzent tragen, wie etwa in úmfahren, aúsgehen, aúfwischen, eínheizen etc. In Fällen wie umfáhren, übersétzen, unterlâufen hingegen trägt, wie auch sonst tendenziell im Deutschen, der lexikalische Kern des Wortes den Akzent. Die Betonung des Präfixes im Typ úmfahren mit dem für Prädetermination typischen fallenden Muster passt im Übrigen zum Faktum der Trennbarkeit dieser Präfixe, die auch vom lexikalischen Kopf isoliert den Hauptakzent tragen: Der Bagger fuhr den Baum úm. In dieser Konstruktion lässt sich erkennen, dass das Präfix in einer zugrunde liegenden syntaktischen Struktur als eine Art Adverb fungiert. In Bildungen des Typs úmfahren/éinfahren und dergleichen hat man es mit Quasikomposita zu tun, innerhalb deren die determinierende erste Konstituente regulär den Ton trägt, so wie in einem herum- oder hereinfahren. In den stammbetonten Ableitungen liegt dem Präfix eher eine präpositionale Funktion zugrunde: Er umfáehrt den Baum $\leftarrow$ Er fährt um den Baum. Der Konflikt ergibt sich im Wortinneren also aus der Tendenz des Deutschen, den jeweiligen lexikalischen Kern zu akzentuieren und andererseits aus dem Prinzip des akzenttragenden Determinans.

Unbetonte prädeterminierende Elemente erzeugen ein jambisches und damit ein typisch postdeterminierendes Akzentmuster, wie in (er) übersétzt/umfáehrt. Bei derartigen Präfixen handelt es sich zunächst (cf. Wells 1990, 254) um regulär akzentuierte Determinantien, wie noch in den erwähnten úebersetzen, úmfahren etc., deren Akzent dann durch den lexikalischen Kopf attrahiert wurde. Eine derartige Entwicklung lässt sich im romanischen Bereich bei der Akzentverschiebung von lat. récipit zu vlat. recípit zu frz. reçoit oder von lat. rétinet $\mathrm{zu}$ vlat. reténet und frz. retient oder von lat. dísplicet $\mathrm{zu}$ vlat. displácet und frz. déplaît (sog. Rekomposition, cf. Rheinfelder 1987 [1953], 11, und Geisler 1982, 57) beobachten. Die Abschwächung determinierender Präfixe könnte als ein Anstoß für den Typwandel von trochäischer OV- zu jambischer VO-Akzentuierung gewertet werden. Das jambische Muster wird freilich darüber hinaus durch verschiedene Faktoren - wie etwa die Thema-Rhema-Progression und die 
Subjekt-Prädikat-Abfolge - unterstützt, so dass es auch innerhalb einer mehr oder weniger vorherrschenden Prädetermination immer seinen Platz hat. ${ }^{6}$

Fallende Akzentmuster innerhalb des Wortes und solche innerhalb syntaktischer Konstruktionen sind differenziert zu betrachten. Im Wortinneren findet sich dieses Betonungsmuster sowohl in eher prädeterminierenden Sprachen wie dem Deutschen oder dem Englischen, wie in Sänger, Kinder, lachend bzw. singer, children, laughing etc., als auch - außer im Französischen - in eher postdeterminierenden Sprachen wie dem Italienischen oder Spanischen: canta, in zweisilbigen Endungen wie in cantate oder in parole sdrucciole wie in cantano oder mangiabile etc., jedenfalls in einer Unmenge von Wörtern mit unbetonter Endsilbe wie cane, porta, orto etc. Solche Endungen und Suffixe fungieren, wie wir gesehen haben, in der Regel als Kopf des Wortes bzw. der Wortform und werden von dem lexikalischen Nukleus determiniert; so auch in Wortbildungen wie pescatore, bufalaio, parlamento und dergleichen, in denen zwar das Suffix aus silbenstrukturellen Gründen den Akzent trägt, aber in sich wieder ein fallendes Muster aufweist. Innerhalb syntaktischer Konstruktionen hingegen haben unbetonte bzw. nicht-betonbare Elemente in enklitischer Postposition meist eine determinierende Funktion als Objekt oder Adverb. Dort ist jedenfalls die Wortfolge postdeterminierend, wie z.B. in ital. farlo, facendolo, fattolo, in imperativischem fammelo! oder auch in andarci und dergleichen. Als unmittelbare Konstituente eines Satzes allerdings werden enklitische Pronomina kaum mehr verwendet, sondern nur noch als Teil eines Satzgliedes, wie eben in farlo, facendolo oder in vistolo als adverbialer Ausdruck, wie in ....vistolo, fu mosso a pietà, keinesfalls jedoch ${ }^{\star} h o$ vistolo. Dies gilt auch für andere romanische Sprachen mit Ausnahme u.a. des Portugiesischen in Sätzen wie Chamo-me Enrique neben Não me chamo Enrique (gemäß Tobler-Mussafia).

Man muss sich nun die Frage stellen, warum Enklitika fast nur noch als Teil eines Satzglieds und speziell als Komplement einer nicht-finiten Verbform vorkommen. Betrachtet man Wortformen wie parlo/parla/parlano etc. und infinite Verbformen mit enklitischem Objektpronomen wie farlo/facendolo/fattolo gegenüber heute inkorrektem (Maria) ${ }^{\star}$ facevalo/ ${ }^{\star}$ fecelo oder auch ${ }^{\star}$ ha fattolo mit finitem Auxiliar, lässt sich festhalten, dass fallender Akzent nur im Wortinneren und in der Syntax eben nur noch in Kombination mit subordinierten nicht-finiten Verbformen auftritt. Man kann nun folgern, dass ein farlo quasi als komplexes Wort behandelt wird und somit einem im Wortinneren möglichen und geläufigen Akzentmuster entspricht, ebenso wie gerundiales facendolo und partizipiales fat-

6 Hubert Haider (2018) meint dazu generell: «Die SVO-Strukturierung ist daher ein Attraktor in der kognitiven Evolution menschlicher Grammatiksysteme». 
tolo sowie auch pensandoci oder ricordarsene. Hier sind Verbum und Pronomen besonders eng miteinander verbunden, so dass Letzteres nach Art einer Endung zu einem Teil des Wortes werden kann (auch durch die Graphie widergespiegelt). In einem *facciolo wäre eine so enge Bindung nicht möglich, da hier das Objektpronomen vom Verbstamm durch eine Subjektendung getrennt ist. Darüber hinaus könnte diese Endung vielfach variieren, also z.B. ^fannolo, `fareilo, *facevalo etc. Das Pronomen wäre hier auch nicht Teil eines Satzglieds, sondern selbst unmittelbares Satzglied, wie übrigens auch in einem heute nicht mehr akzeptablen *ho fattolo, in dem das Pronomen nicht nur die Partizipform determinierte, sondern das ganze Prädikat ho fatto, wie in korrektem l'ho fatto.

${ }^{*}$ Facciolo wäre also nicht so etwas wie ein komplexes Wort sondern eine syntaktische Konstruktion, ein zweigliedriger vollständiger Satz mit zwei Vorgangsbeteiligten, wie lo faccio, während die Enklitika, wie gesagt, heute nur noch als Teile von untergeordneten Satzgliedern auftreten. Vorangestellte Elemente wie das Objektpronomen im letzten Beispiel sind grundsätzlich weniger eng mit ihrer Bezugseinheit verbunden als nachgestellte (cf. Wandruszka 1992 zur sog. «Suffixpräferenz»), zumal sie erst sekundär in Anteposition gelangen, während das den infiniten Verbformen folgende Pronomen, historisch betrachtet, einfach dort stehen bleibt und auch phonetisch eng mit der Verbform verschmelzen kann, wie speziell in farlo, das im Altitalienischen sogar assimilierte Varianten wie fallo kennt. Hinzukommt, dass das finite jambische lo faccio einer Thema-Rhema-Progression folgt, die in nicht-satzhaltigen infiniten Formen wie farlo oder facendolo keine wesentliche Rolle spielt. Demnach sollte ein vollständiger Satz im Italienischen mit einem akzenttragenden Wort im definierten Sinn enden und nicht mit einem unbetonbaren unmittelbaren Satzglied wie in ${ }^{\star}$ facciolo. Die italienische Syntax zeigt sich auch in diesem Fall jambisch strukturiert, im Unterschied zu einem altital. fecelo 'er machte es'.

Wie im Französischen erfährt im Italienischen auch der Imperativ eine gesonderte Behandlung, wenn das Verbum in Spitzenposition gebracht werden soll und das Pronomen daher enklitisch wird bzw. bleibt. Dies wird gleichfalls durch die Annahme erklärlich, dass Konstruktionen wie prendilo! und scrivetelo! oder vacci! 'geh hin!' als komplexe Wörter betrachtet werden, worauf im Übrigen auch die Zusammenschreibung hindeutet (cf. auch das infinitivische non prenderlo!). Eine Konstruktion wie prendilo! präsentiert sich nach Form und Akzentmuster wie etwa das proparoxytone Nomen cóndilo 'Gelenkkopf' bzw. wie die dreisilbige Verbform prendono. Wenn im Französischen und im Italienischen bei solchen Ausdrücken die Postposition des Pronomens bewahrt werden kann, weil die Konstruktion als ein Wort gesehen wird, muss im Französischen das Enklitikon akzentuiert werden, prends-lé, während im Italienischen das Pronomen enklitisch unbetont bleiben kann. Das heißt, frz. prends-lé! ist endbetont wie ein zweisilbi- 
ges französisches Wort und ital. prendilo ist initialbetont wie ein einfacher italienischer Dreisilber.

\section{Das mot phonique}

Das Phänomen des mot phonique hat wesentlich mit der Generalisierung des französischen Wortakzents auf der letzten Silbe zu tun. Das einzelne Wort kann hier seinen Akzent weitgehend aufgeben, weil es keinen individuell unterschiedlichen silbenstrukturell oder morphosemantisch motivierten Eigenakzent besitzt. Das heißt, jedes Wort hat einen vorhersehbaren Akzent, der in einem vorhersehbaren gleichgerichteten Wortgruppenakzent aufgehen kann, was eben genau dann geschieht, wenn alle Wörter im Prinzip auf der gleichen Silbe betont werden und zwar auf der letzten. Die Satzglied- oder Wortgruppenbetonung hat mit der letzten Silbe einen übergreifenden Flucht- oder Zielpunkt, auf den die gespannte Intonation zuläuft und der einen markanten Abschluss bildet. Es ist also nicht nur das letzte Wort, wie im Italienischen und anderen postdeterminierenden Sprachen, sondern gerade die letzte Silbe, die den Satz(glied)-Akzent trägt, was perfekt der natürlichen Tendenz zur Betonung des Endes einer Äußerung gemäß einer unmarkierten Thema-Rhema-Progression entspricht - wenngleich im Französischen die natürliche Übereinstimmung zwischen Rhematizität und internem Wortakzent, wie gezeigt, nachhaltig gestört ist: Der Wortakzent verläuft parallel zum Satzgruppenakzent jambisch steigend, während die innere Struktur polymorphematischer Wörter eben nicht postdeterminierend ist.

In einer Sprache hingegen, in der die Wörter nicht nur auf der letzten sondern auch auf der vorletzten oder vorvorletzten Silbe betont sein können, kann eine Wortgruppe nicht prinzipiell das gleiche Akzentmuster aufweisen wie die darin enthaltenen einzelnen Wörter. Die letzte Silbe eines Wortes ist immer die letzte, während die erste Silbe eines Wortes die letzte (a), die vorletzte (b) oder die vorvorletzte (c) sein kann:

(a) arbeitet mit Fléiß, (b) ... mit Fréude, (c) ... mit Âusdauer

Das mot phonique könnte also selbst in einer Sprache mit durchgehendem Initialakzent nicht immer mit dem gleichen Betonungsmuster enden wie die einzelnen vorausgehenden Wörter mit ihrer je unterschiedlichen Akzentposition. Wenn die Initialbetonung nicht durchgängig ist - man denke etwa an ein deutsches ... arbeitet mit Verstând - kann es ohnehin zu keiner völligen Übereinstimmung zwischen Wort und Wortgruppe kommen. Bei durchgehender Oxytonie hingegen endet im Prinzip jedes mot phonique so wie die einzelnen darin enthaltenen Wörter auf eine akzentuierte Silbe. Ein wesentliches Charakteristikum des mot pho- 
nique ist darin zu sehen, dass durch die akzentuierte Endsilbe das Ende der Wortgruppe eindeutig markiert wird, während mit einer unbetonten Endsilbe der Intonationsbogen nicht mit dieser Deutlichkeit geschlossen werden könnte. Das Ende ist deutlich wahrnehmbar, weil sich die akzentuierte letzte Silbe von allen vorangehenden nicht-betonten Silben abhebt, während sich die letzte Silbe bei fallendem Akzent nicht von allen ihr vorausgehenden, sondern im Prinzip nur von der allerersten betonten Silbe unterscheiden würde.

Um die oft als «schwebend» bezeichnete Intonation innerhalb des französischen mot phonique herzustellen, müssen die Wortakzente, außer dem letzten, nivelliert, d.h. abgesenkt werden. Die betonte Silbe eines Wortes wird an die jeweils vorangehende nicht (mehr) betonte Silbe angeglichen oder jedenfalls angenähert. Diese Silben sind dann von etwa gleicher Länge, Höhe und Intensität (cf. Meunier 2017, 34), im Unterschied zur jeweils letzten Silbe am Ende des mot phonique, wie z.B. in dem Satz: Maman a perdu une dent, wobei so etwas wie ein überlanger Anapäst mit betonter und gelängter Schlusssilbe entsteht ( $\left.\cup \cup \cup^{\prime}-\right)$. Bei trochäisch fallender Akzentuierung hingegen können akzentuierte Silben nicht ohne weiteres an vorangehende nachtonige Silben angeglichen werden, wie etwa in dem Satz Peter wollte Schuhe kaufen. Hier müsste die erste Silbe von wollte so schwach betont sein wie die zweite Silbe von Peter (mit ihrem Schwa) und dann auch wie die zweite Silbe von wollte selbst. Entsprechend müssten die beiden Silben von Schuhe gleich betont bzw. unbetont und jedenfalls gleichgewichtig sein, wie auch die von kaufen. Dies stellt vor allem deshalb ein Problem dar, weil die Differenz zwischen akzentuierter und nachtoniger Silbe im Deutschen größer ist als die zwischen vortoniger und akzentuierter Silbe. Wenn nun diese Differenz zwischen haupttoniger Silbe, wie eben in wóllte oder Schúhe, und besonders schwacher nachfolgender Silbe nivelliert wird und letztere so ihre charakteristische Nachtonigkeit verliert, entsteht durch einen akustischen Kippeffekt ein tendenziell jambisches Akzentmuster, also in etwa wólltè oder Schúhè. Durch die Deakzentuierung der Haupttonsilbe nähert sich diese der unbetonten Silbe an (und umgekehrt), wobei im Deutschen auch ein schwacher Nebenakzent, etwa in einem Schwímmèr als unangemessene Akzentuierung des Suffixes empfunden wird. Solange die letzte Silbe schwach betont oder jedenfalls nicht wirklich unbetont ist und allgemein, wenn zwei aufeinanderfolgende Silben akzentuell annähernd gleichwertig sind, entsteht tendenziell ein Jambus, weil eine nicht deutlich abgesenkte Nachtonsilbe zu viel Gewicht relativ zur Tonsilbe erhält und so den genannten Kippeffekt auslöst.

Auf vortonigen Silben hingegen, die normalerweise ebenfalls nicht oder nur sehr schwach betont sind, ist ein Nebenakzent nicht nur eher möglich, sondern gerade im Französischen durchaus geläufig, wie z.B. in den von Meisenburg/Selig $(2008,159)$ erwähnten bàteau-citérne, la màjeure partíe, àcculturatión u.a. Die- 
ser auch als contre-accent bezeichnete Initialakzent tritt insbesondere in silbenzählenden Sprachen wie dem Französischen auf, in denen der Unterschied zwischen akzentuierter und nicht akzentuierter Silbe nicht so ausgeprägt ist wie im akzentzählenden Deutschen. Dies führt zu der angesprochenen «schwebenden» Betonung/Intonation des Französischen, die sich aus der Gleichgewichtigkeit der Silben ergibt und die Bildung von mots phoniques befördert. Deutsche Sprecher neigen interessanterweise dazu, die letzte Silbe französischer Wörter zuungunsten der jeweils vorangehenden besonders nachdrücklich $\mathrm{zu}$ betonen, wodurch wie im Deutschen wieder ein starker Wortakzent produziert wird.

\section{Schlussbemerkung}

Formale signifikantenseitige Entwicklungen, hervorgerufen durch äußere Einflüsse oder einfach bedingt durch die Natur des sprachlichen Mediums, können dysfunktionale Modifikationen der inneren Struktur der Sprache zur Folge haben. Es kann sich eine funktionale Entkoppelung von Form und Inhalt vollziehen, ohne dass ein solcher mismatch vom Sprachbenutzer wahrgenommen und als besonders störend empfunden werden muss. Autokorrektur und «Selbstheilungskräfte» der Sprache nach der Vorgabe form follows function sind nicht immer stark genug, um derartige Störungen des Zusammenspiels der verschiedenen Ebenen der Sprache zu verhindern oder zu beheben. Das Instrument Sprache funktioniert offenbar auch bei suboptimaler oder dysfunktionaler Ausdrucksgestaltung, da es prinzipiell auch durch den (lexikalischen) Inhalt der Zeichen gestützt wird, der die passende Interpretation eines komplexen Ausdrucks gleichwohl ermöglicht. Schlagende Beispiele hierfür sind die nachgestellten betonten Pronomina, in frz. vient-il, donne-le-lui, vas-y und dergleichen, oder die oxytonen Wortformen wie chanter, chanta, chanteur etc. als Ergebnis des Verstummens der lateinischen Nachtonsilben. Dies ist umso bemerkenswerter, als das Phänomen im Französischen keineswegs nur vereinzelt sondern als Grundprinzip quasi ausnahmslos auftritt, was dazu führt, dass das System an dieser Stelle regelrecht kippt und einen massiven Typwandel erfährt, hier in Form des Verlustes fallender trochäischer Akzentmuster und ihrer Ersetzung durch jambische.

Diese nicht funktional-inhaltlich motivierte Veränderung auf der Signifikantenseite zieht im Wortinneren keine dem jambischen Akzentmuster entsprechende postdeterminierende Organisation nach sich, in der das jeweilige Determinans betont wäre; denn der Akzent kommt in der Regel auf die betonte Silbe eines ursprünglich mehrsilbigen Funktorkopfs zu stehen und damit prinzipiell auf das dann einsilbige Flexiv oder Suffix wie in cantavit $\rightarrow$ chanta oder ligamentum $\rightarrow$ ligament usw. Die Endbetonung der Wörter und Wortformen passt formal zur 
postdeterminierenden Struktur der französischen Syntax, nicht jedoch die Kopffunktion der betonten Silbe. Solche systematischen Verwerfungen ergeben sich in unserem Fall daraus, dass durch die lineare Bewegung der Sprachproduktion positionsbedingte Asymmetrien in der Akzentstärke entstehen. So ist die Nachtonsilbe in der Regel noch schwächer als die Vortonsilbe, weshalb sie zu Reduktion oder Tilgung tendiert, gewissermaßen im Windschatten des Hauptakzents und zur Erhaltung der Gesamtenergie der beiden unmittelbar aufeinanderfolgenden Silben. Da die Nachtonsilben am Ende des Wortes normalerweise die Flexive und Suffixe enthalten, die als Köpfe des Ausdrucks fungieren, bedeutet ihr Verstummen den Verlust des morphologischen Kopfes, jedenfalls des unbetonten Teils eines mehrsilbigen Kopfes. Identifiziert man den Kopf lateinischer Wörter mit der am Wortende befindlichen Konstituente, die unbetont ist oder jedenfalls diese unbetonte Konstituente enthält, dann besitzen die französischen Entsprechungen keinen derartigen Kopf (mehr). Was bleibt, ist der von uns als mismatch bezeichnete betonte Kopf oxytoner Wortformen, wie in chantons, nageur oder barrage, fragile und generell im Französischen. Wenn der Kopf im Lateinischen einsilbig war, wird die französische Nachfolgeform morphologisch vollständig «kopflos» wie in vient $\leftarrow$ venit bzw. in Nomina wie chant $\leftarrow$ cantus, fils $\leftarrow$ filius oder einem Adjektiv wie las $\leftarrow$ lassus, die nur aus dem betonten ursprünglichen Komplement bestehen. Immerhin trägt in diesem Fall nicht der lateinische Kopf, sondern das einstige Komplement den Akzent.

\section{Bibliographie}

Baldinger, Kurt, Post- und Prädeterminierung im Französischen, in: id. (ed.), Festschrift Walther von Wartburg, vol. 1, Tübingen, Niemeyer, 1968, 86-106.

Barme, Stefan, Gesprochenes Französisch, Berlin/Boston, De Gruyter, 2012.

Eckert, Gabriele, Sprachtypus und Geschichte. Untersuchungen zum typologischen Wandel des Französischen, Tübingen, Narr, 1986.

Eisenberg, Peter, Grundriss der deutschen Grammatik, Stuttgart, Metzler, ${ }^{4} 2013$.

Geisler, Hans, Studien zur typologischen Entwicklung. Lateinisch - Altfranzösisch · Neufranzösisch, München, Fink, 1982.

Grevisse, Maurice, Le bon usage. Grammaire française, Gembloux, Duculot, ${ }^{10} 1975$.

Guiraud, Pierre, La syntaxe du français, Paris, Presses Universitaires de France, ${ }^{6} 1980$.

Haider, Hubert, Endstation SVO? Warum viele Sprachen zu SVO-Sprachen wurden und viele angebliche «SVO»-Sprachen keine sind, Berlin, Humboldt-Universität, 2018, <https://www. uni-salzburg.at/fileadmin/multimedia/Linguistik/2018-Feb-HUB-Berlin-HA.pdf> [letzter Zugriff 30.07.2019].

Kaschny, Christine, Primärer Wortakzent und morphologische Struktur im Italienischen. Eine theoriebezogene, quantitativ und empirisch angelegte Untersuchung, Konstanz, Bibliothek der Universität Konstanz, 2011. 
Meisenburg, Trudel/Maria Selig, Phonetik und Phonologie des Französischen, Stuttgart, Klett, 2008.

Meunier, Christian, Grammaire participative: enseignée avec la participation active des apprenants, Marseille, Les Editions du FLE, 2017.

Pomino, Natascha/Stark, Elisabeth (edd.), Proceedings of the V Nereus International Workshop on «Mismatches in Romance», Universität Konstanz, Arbeitspapier Nr. 125, Fachbereich Sprachwissenschaft, 2011.

Rheinfelder, Hans, Altfranzösische Grammatik, vol. 1: Lautlehre, München, Hueber, ${ }^{5} 1987$ [1953].

Sasse, Hans-Jürgen, Gedanken über Wortstellungsveränderung, Papiere zur Linguistik 13:14 (1977), 82-142.

Wandruszka, Ulrich, Post- oder Prädetermination in den romanischen Sprachen, Romanistisches Jahrbuch 31 (1980), 56-72.

Wandruszka, Ulrich, Klassemisch versus lexemisch. Zwei Grundtypen sprachlicher Strukturbildung, Papiere zur Linguistik 41:2 (1989), 77-100.

Wandruszka, Ulrich, Zur Suffixpräferenz. Prolegomena zu einer Theorie der morphologischen Abgeschlossenheit, Papiere zur Linguistik 46:1 (1992), 3-27.

Wandruszka, Ulrich, Figure et fond en morphosyntaxe et la notion d'importance, in: Dufter, Andreas/Jacob, Daniel (edd.), Syntaxe, structure informationnelle et organisation du discours dans les langues romanes, Bern/Berlin, Lang, 2011, 173-188.

Wandruszka, Ulrich, Sprache - linearisierte Struktur in Bewegung. Eine Einführung in die Mechanik der Sprache auf der Basis der Kategorialgrammatik, Tübingen, Stauffenburg, 2015.

Weinrich, Harald, La place de l'adjectif en français, Vox Romanica 25 (1966), 82-89.

Weinrich, Harald, Sprache in Texten, Stuttgart, Klett, 1976.

Wells, Christopher, J., Deutsch. Eine Sprachgeschichte bis 1945, Tübingen, Niemeyer, 1991.

Wunderli, Peter/Benthin, Karola/Karasch, Angela, Französische Intonationsforschung. Kritische Bilanz und Versuch einer Synthese, Tübingen, Narr, 1978.

Wunderli, Peter, Intonationsforschung und Prosodie, in: Holtus, Günter/Metzeltin, Michael/ Schmitt, Christian (edd.), Lexikon der Romanistischen Linguistik (LRL), vol. 5/1: Französisch, Tübingen, Niemeyer, 1990, 34-46. 